\title{
artigo
}

Almeida, J.M.C.; Rolim, I.L.T.P.; Penha, J.S.; Serra, E.B.; Lopes, G.S.G.; Palmeira, C.R.;

Folder educativo: vivenciando a ética profissional e compromisso social durante a pandemia de COVID-19l

\section{Folder educativo: vivenciando a ética profissional e compromisso social durante a pandemia de COVID-19}

\author{
Educational folder: experiencing professional ethics and social commitment during the COVID-19 pandemic \\ Carpeta educativa: experimentando la ética profesional y el compromiso social durante la pandemia del COVID-19
}

\begin{abstract}
RESUMO
Objetivo: descrever o alcance de competência ética e compromisso social de discentes durante a confecção de material educativo na pandemia da COVID-19. Método: relato de experiência de discentes de um grupo de pesquisa na construção de folder educativo, no período de abril a maio de 2020. 0 trabalho foi dividido em etapas e subgrupos, discutindo-se a abordagem geral da diabetes mellitus (DM) e relação com a COVID-19, educação em saúde e contribuição para promoção da saúde de diabéticos. Resultados: elaborou-se o folder "Diabetes e Imunidade: cuidados adicionais em tempos de COVID-19", divulgado em mídia on-line. Os envolvidos participaram em forma de debate, reflexão, cooperação, interação e compromisso social. Conclusões: promover educação em saúde de maneira on-line possibilitou ao grupo vivenciar a formação moral e compromisso social, além de uma abordagem diferente do cuidado, com discussões sobre o papel do discente, docente, universidade e sociedade.
\end{abstract}

DESCRITORES: Ética profissional; Educação em saúde; Enfermagem; Covid-19; Isolamento social;

\section{ABSTRACT}

Objective: to describe the reach of ethical competence and social commitment of students during the preparation of educational material in the pandemic of COVID-19. Method: experience report of students from a research group in the construction of an educational folder, from April to May 2020. The work was divided into stages and subgroups, discussing the general approach to diabetes mellitus (DM) and its relationship with COVID-19, health education and contribution to the health promotion of diabetics. Results: The folder "Diabetes and Immunity: additional care in times of COVID-19" was elaborated, published in online media. Those involved participated in the form of debate, reflection, cooperation, interaction and social commitment. Conclusions: promoting health education online enabled the group to experience moral education and social commitment, in addition to a different approach to care, with discussions on the role of students, teachers, universities and society.

DESCRIPTORS: Ethics professional; Health education; Nursing; Covid-19; Social isolation;

\section{RESUMEN}

Objetivo: describir el alcance de la competencia ética y el compromiso social de los estudiantes durante la elaboración de material educativo en la pandemia de COVID-19. Método: relato de experiencia de estudiantes de un grupo de investigación en la construcción de una carpeta educativa, de abril a mayo de 2020. El trabajo se dividió en etapas y subgrupos, discutiendo el abordaje general de la diabetes mellitus (DM) y su relación con COVID-19, educación para la salud y contribución a la promoción de la salud de los diabéticos. Resultados: se elaboró la carpeta "Diabetes e inmunidad: cuidados adicionales en tiempos de COVID-19", publicada en medios online. Los involucrados participaron en forma de debate, reflexión, cooperación, interacción y compromiso social. Conclusiones: promover la educación para la salud en línea permitió al grupo experimentar la educación moral y el compromiso social, además de un enfoque diferente del cuidado, con discusiones sobre el rol de los estudiantes, docentes, universidades y sociedad.

DESCRIPTORES: Ética professional; Educación en salud; Enfermería; Covid-19; Aislamiento social;

RECEBIDO EM: 27/01/2021 APROVADO EM: 10/02/2021

\section{Jocelha Maria Costa de Almeida}

Enfermeira do Hospital Municipal Djalma Marques. Especialista em Saúde da Família e Unidade de Terapia Intensiva. Discente do Mestrado Acadêmico em Enfermagem da Universidade Federal do Maranhão.

ORCID: 0000-0002-3147-954X 


\section{Isaura Letícia Tavares Palmeira Rolim}

Enfermeira. Professora da Universidade Federal do Maranhão. Mestrado e Doutorado em Enfermagem pela Universidade Federal do Ceará.

ORCID: 0000-0002-8453-2543

\section{Jaiza Sousa Penha}

Enfermeira. Especialista em Saúde da Mulher. Discente do Mestrado Acadêmico em Enfermagem da Universidade Federal do Maranhão. ORCID: 0000-0001-9805-3802

\section{Eliana Brugin Serra}

Enfermeira da Rede Sarah de Hospitais do Aparelho Locomotor. Especialista em Unidade de Terapia Intensiva. Discente do Mestrado Acadêmico em Enfermagem da Universidade Federal do Maranhão.

ORCID: 0000-0003-2295-4115

\section{Geysa Santos Góis Lopes}

Enfermeira da Rede Sarah de Hospitais do Aparelho Locomotor. Especialista em Enfermagem do Trabalho com Habilitação em Docência Superior. Mestre em Enfermagem. Doutoranda do Programa Doutoral em Ciência da Enfermagem da Universidade do Porto. ORCID: 0000-0002-6801-1940

\section{Cindy Rebouças Palmeira}

Arquiteta, Professora da Uniateneu Centro Universitário. Especialista em Arquitetura de Interiores. Mestre em Ciências da Cidade. ORCID: 0000-0002-6757-723X

\section{INTRODUÇÃO}

A graduação na área da saúde enfrenta transformações conceituais e metodológicas para o desenvolvimento de ensino-aprendizagem com o propósito de atender às demandas de saúde em acelerada evolução e às novas diretrizes no ensino, além de conduzir o discente ao protagonismo, e o docente ao papel de facilitador. Neste contexto, as ferramentas tecnológicas de ensino, aplicadas à luz de teorias pedagógicas, promovem mudanças nesse processo $^{1,2,3}$.

A tecnologia para o ensino é entendida como o meio pelo qual é possível reproduzir, modificar, acrescentar e estabelecer novas relações com o processo ensino-aprendizagem. Também pode ser conceituada como um grupamento de técnicas e processos para determinado fim ${ }^{4}$.

Em saúde, a formação de recurso humano requer um profissional que alcance a interdisciplinaridade na tomada de decisão, liderança, interlocução, raciocínio crítico-reflexivo e que tenha comprometimento ético e social com a população $0^{5,2}$.

O compromisso social é elemento indispensável na formação de recursos humanos em saúde, possibilitando a transformação de particularidades determinantes da saúde das populaçóes, principalmente dos grupos em situações de vulnerabilidade, como os pacientes portadores de Diabetes Mellitus (DM). Neste relato, compromisso social é entendido como um processo de conhecimento da realidade social, das necessidades da população, levando os profissionais a desenvolverem práticas contextualizadas, percebendo as particularidades culturais dos indivíduos, contribuindo para a transformação das condicionantes de vida na busca da dignidade ${ }^{6}$.

Estudo prévio aponta que, por vezes, durante o processo de formação em saúde, o ensinamento da ética e do compromisso social aparecem de modo isolado do ambiente pedagógico, afastando o aluno desses aspectos. Consequentemente, alguns impeditivos organizacionais e relacionados ao corpo docente e discente foram identificados, como pouco conhecimento, falta de habilidade para ensino dessas questões, ausência de recursos, falhas no processo de aprendizagem e escassez de facilitadores?

Assim, este estudo buscou responder a seguinte pergunta norteadora: De que forma um grupo de pesquisa pode desenvolver competência ética e compromisso social no cuidado de pacientes diabéticos durante a pandemia de Covid-19?
Dessa forma, este relato objetivou descrever a experiência da construção de um material educativo em saúde como ferramenta para alcance de competência ética e compromisso social no processo de formação em saúde. É válido destacar que as ações e práticas do ensino nessa área se propõem a ultrapassar as barreiras das universidades, alcançando os cidadãos com a oferta de instrumentos que contribuam para a promoção da saúde e prevenção de agravos, melhorando esses indicadores.

\section{MÉTODO}

Estudo descritivo, do tipo relato de experiência, resultante da necessidade de refletir sobre a abordagem pedagógica e teórico-metodológica de um grupo de estudo e pesquisa universitário quanto ao desenvolvimento de competência ética e compromisso social no contexto da COVID-19.

A discussão acerca do tema surgiu da necessidade mundial de isolamento social e consequente afastamento do grupo de pesquisa de sua clientela. O grupo compreendeu que as ações coletivas poderiam ser suspensas juntamente com as aulas presenciais de uma universidade pública do nordeste brasileiro. No entanto, decidiu-se manter as reunióes on-line, onde 
foi deliberado a construção de material educativo para portadores de DM. Com clareza de conteúdo, os materiais tiveram o objetivo de abordar a pandemia, orientar a população, informar sobre riscos, cuidados especiais e promover o apoio mútuo.

Participaram desta construção duas professoras da universidade, quatro alunos da graduação, duas pós-graduandos stricto sensu e uma pesquisadora. $\mathrm{O}$ trabalho foi dividido em categorias, incluindo abordagem geral sobre DM e a sua relação com a COVID-19, educação em saúde e contribuição para a promoção da saúde dos portadores desse agravo. $\mathrm{O}$ processo de elaboração do material educativo se deu durante um período de quarentena, entre os meses de abril e maio de 2020.

A dinâmica teve como base teórica a Metodologia da Problematização de Paulo Freire, que está ancorada na compreensão da realidade para nela intervir e transformar a partir de três etapas: detectar o problema, busca de explicação e proposta de solução ${ }^{8}$. A problematização, na visão de Paulo Freire, aponta uma imersão crítica na realidade da sociedade, a fim de subtrair entendimento que leva ao aprendizado, pois na ação-reflexão-ação são construídos os conhecimentos considerando os determinantes contextuais.

Assim, é a reflexão do homem sobre si, integrado ao seu contexto, que permite a construção do ser sujeito, à medida em que se compromete, tomando consciência de sua realidade ${ }^{9}$. Portanto, uma atividade que quer despertar na população atitude crítica, precisa trazer o universo temático do povo, ou seja, seus temas geradores. A pedagogia de Paulo Freire instrumentaliza docente e discente para o novo, para além dos limites impostos, leva a uma pedagogia ética, onde o outro é levado em consideração na tomada de decisões, além de abrir caminhos para uma sociedade mais humanizada ${ }^{10}$.

\section{RELATO DE EXPERIÊNCIA}

As atividades do grupo de pesquisa foram construídas a partir da distribuição de tarefas entre os integrantes. Inicialmente, ocorreram discussões sobre os conteúdos didáticos, imagens e escolha da informação a ser divulgada. O senso de equipe e colaboração foi trabalhado pelas mentoras como requisito para a aquisição de confiança, conhecimento e das habilidades a serem alcançadas, atingindo o propósito da vivência ética e compromisso social do grupo. Os envolvidos demonstraram empenho ao expor seus pontos de vista, dúvidas e anseios. Essa preocupação refletiu na escolha criteriosa da linguagem e ilustrações que compuseram o material, bem como de que forma seria o alcance do público-alvo.

\section{O trabalho foi}

dividido em

categorias, incluindo

abordagem geral

sobre DM e a

sua relação com

a COVID-19,

educação em saúde e

contribuição para a

promoção da saúde

dos portadores

desse agravo.

Assim, na etapa 1 do processo, denominada "Como cuidar do DM em tempos de COVID-19?”, os graduandos estudaram os temas: transmissão e desenvolvimento de formas graves da COVID-19 em popula- ções especiais, como os diabéticos; orientações para o bem-estar; uso e continuidade das medicações prévias; formas de evitar o contágio e dicas de como encontrar apoio familiar e social em tempos de COVID-19. Neste momento, o grupo 1 teve liberdade para elaborar os textos e temas a partir do objetivo apontado: produzir um material educativo para diabéticos em tempos de pandemia pela COVID-19.

$\mathrm{Na}$ etapa 2, "Arquitetura de informações", o objetivo foi o aprimoramento do documento 1 , em que as mestrandas refletiram, pesquisaram e reorganizaram as informações. De forma a garantir a cientificidade do conteúdo, foram revisados e estudados manuais do Ministério da Saúde e consensos da Sociedade Brasileira de Diabetes. O material foi organizado em blocos: informações gerais sobre $\mathrm{CO}$ VID-19, recomendações para prevenção do contágio, com destaque para a alimentação saudável, atividade física, controle da glicemia capilar e uso contínuo e correto das medicações.

Por conseguinte, na etapa 3, "Oficina: Como criar um folder para os pacientes portadores de DM", os docentes e a pesquisadora arguiram as fontes de informações e a redação que resultaram no documento 3. Assim, na etapa 4, "Elaboração do folder", foram selecionadas as figuras mais adequadas, a fim de encontrar as melhores representações gráficas de domínio público, tamanho de fontes e integração de texto e ilustrações para arte definitiva. Após quatro horas de trabalho on-line foi construído o folder educativo "Diabetes e Imunidade: cuidados adicionais em tempos de COVID-19" (Figura 1).

Finalmente, o material foi apreciado pelos membros do grupo de estudo. Após aprovação, todos se comprometeram a fazer a divulgação. A execução do processo educativo e todas as etapas foram realizadas de forma participativa. A condução adotada promoveu interação e compromisso dos alunos através de uma ponderação dialogada, onde percebeu-se que os discentes se envolveram numa reflexão ética referente a práxis da responsabilidade sobre o cuidado com o outro. 


\section{DISCUSSÃO}

Segundo Paulo Freire, o compromisso social parte de uma realidade concreta para a tomada de decisão segundo um plano de ação por parte do sujeito/ator social. Isso quer dizer que o agir e o refletir é a primeira condição para que uma pessoa possa assumir um ato comprometido ${ }^{11}$.

O compromisso social do discente consiste em tentar desenvolver a formação humana para transformar o indivíduo em cidadão consciente e atuante na promoção da sua saúde. Para tanto, a sua formação deve ser competente, técnica e, acima de tudo, ética, com valores humanísticos. Além disso, o compromisso social induz ao comportar-se socialmente, envolvendo a ética e a competência do enfermeiro, tanto na docência, quanto na prática assistencial, sendo suas atitudes produzidas por uma estrutura socialmente construída ${ }^{12}$.

Trabalhar um folder educativo de maneira remota compreende uma demanda social e mostra-se como importante instrumento no contexto de educação em saúde, constituindo uma forma de cuidado que favorece o desenvolvimento da ética profissional. Estabeleceu-se inúmeras reflexões, debates, aprendizagem e um olhar mais atento das informações veiculadas. Cabe ao docente apontar o caminho para uma prática consciente, segura e ética ${ }^{13}$.

Nesse contexto, uma pesquisa que objetivou validar uma cartilha educativa sobre a prevenção de dengue, apontou que a enfermagem necessita expandir e valorizar as suas produções, não sendo estas necessariamente recursos tecnológicos de ponta ou de alto custo, como artefatos e inventos, mas sim de estratégias para dinamizar o processo de trabalho ou materiais didático-pedagógicos como ferramentas para educar e promover saú$\mathrm{de}^{14}$. Ademais, no cenário de atuação da

\section{Figura 1- Folder educativo - Diabetes e Imunidade: Cuidados adicionais em tempos de COVID-19.}
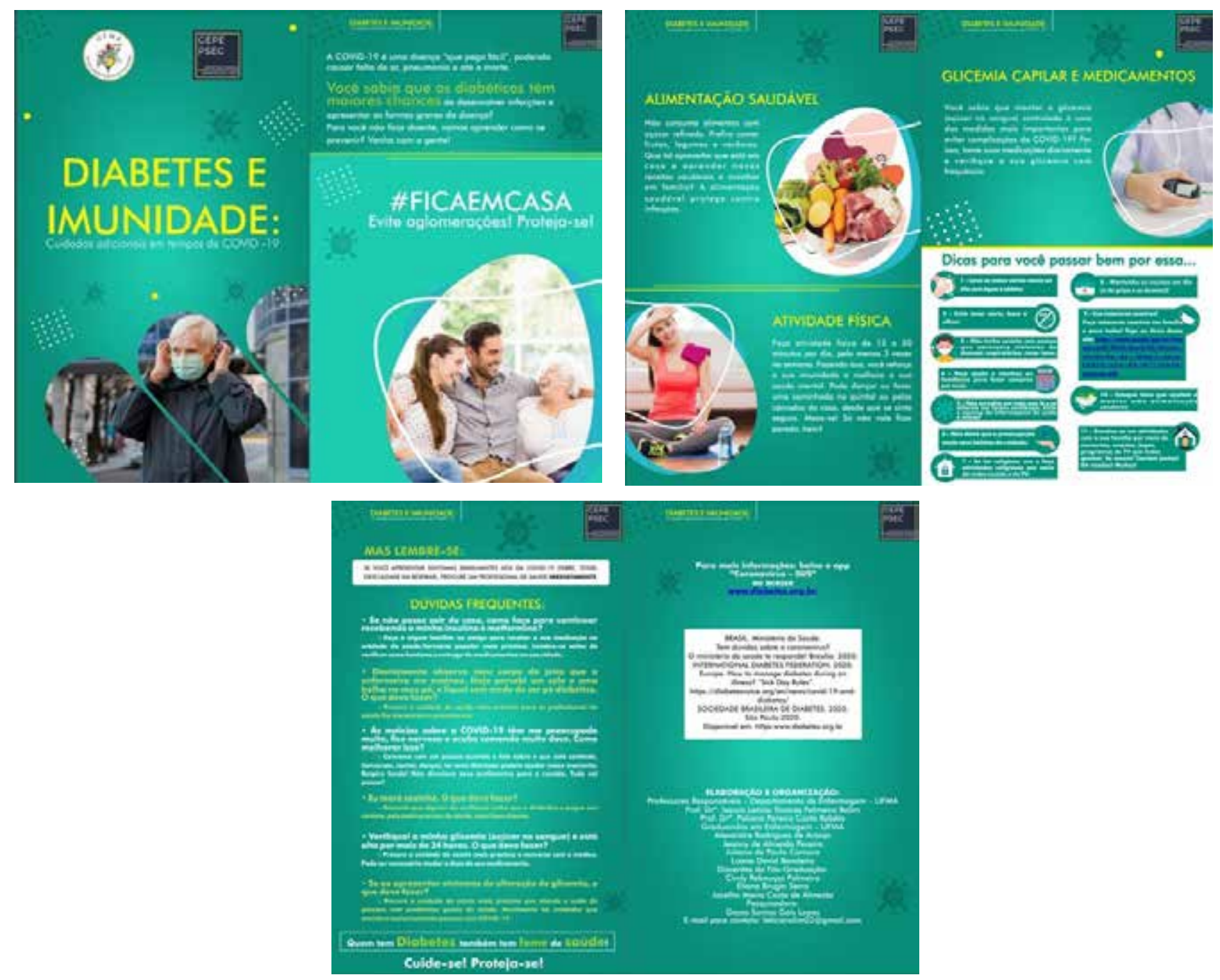

FONTE: Autores. 


\section{artigo}

Enfermagem, o cuidar e o educar não estão dissociados. Ao contrário, em sua prática de cuidar, o enfermeiro também pode educar, de forma concomitante, considerando a ética e compromisso enquanto profissional de saúde ${ }^{15}$.

Contextualizando, ressalta-se que a universidade no Brasil é considerada um lugar de aprendizado e, sobretudo, de transmissão de conhecimento ${ }^{16}$. Contudo, essas instituições têm passado por uma fase de transformação, atravessando uma crise de legitimidade e questionamentos sobre seu papel na produção de conhecimentos e formação de profissionais com adequado perfil às necessidades da população. Nesse sentido, fomentar a dimensão ética ainda é um desafio em muitas instituições de ensino superior ${ }^{17}$.
Apesar disso, esses estabelecimentos educacionais estão envolvidos na cultura e desenvolvimento de ações que promovam o compromisso social e suas manifestações, que tendem a assegurar as diversas atuações dessas instituições na sociedade, objetivando o reconhecimento e valorização ${ }^{18}$.

\section{CONCLUSÃO}

A oportunidade de trabalhar educação em saúde de forma remota pela imposição da pandemia da COVID-19 e a necessidade do distanciamento entre as pessoas, mostrou-se uma tática na construção de conhecimento e fortalecimento da ética e compromisso social do grupo de pesquisa.

Observou-se que a atividade foi satisfatória e inédita para o grupo, pois a es- tratégia utilizada promoveu a construção de conhecimento, despertou no discente o interesse na busca de soluções criativas de baixo dispêndio e trouxe a possibilidade de alcançar grande número de pessoas através das redes sociais e sites amplamente conhecidos.

Acredita-se que a estratégia da elaboração de uma ferramenta educativa construída com base na problematização num contexto de pleno isolamento social, levou os docentes a apoiarem os graduandos na construção e retomada de comportamentos, atitudes, valores e crenças, pautados no bem estar da população, em especial os diabéticos, não só nos saberes apreendidos nas diversas disciplinas acadêmicas, mas acima de tudo, no compromisso social e na ética profissional. -

\section{REFERÊNCIAS}

1. Janicas RCSV, Narchi NZ. Evaluation of nursing student's learning using realistic scenarios with and without debriefing. Rev Latino-Am. Enfermagem 2019;27:1-9.

2. Salvador PTCO, Rodrigues CCFM, Lima KYN, Alves KYAA, Santos VEP. Uso e desenvolvimento de tecnologias para o ensino apresentados em pesquisas de enfermagem. Rev Rene 2015;16(3):442-450.

3. Weeks KW, Coben PD, O'Neill D, Jones A, Weeks A, Brown M, et al. Developing and integrating nursing competence through authentic technology-enhanced clinical simulation education: Pedagogies for reconceptualising the theory-practice gap. Nurse Educ Pract. 2019;37:29-38.

4. Kenski VM. Educação e tecnologias: o novo ritmo da informação. Campinas: Papirus, 2007.

5. Yeun EJ, Bang HY, Ryoo EN, Ha EH. Attitudes towards simulation- based learning in nursing students: an application of Q methodology. Nurse Educ Today 2014;34(7):1062-1068.

6. Nesje K. Personality and professional commitment of students in nursing, social work, and teaching: a comparative survey. Int J Nurs Stud. 2016;53:173-181.

7. Sollami A, Caricati L, Mancini T. Does the readiness for interprofessional education reflect students' dominance orientation and professional commitment? Evidence from a sample of nursing students. Nurse Educ Today 2018;68:141-145.

8. Mizukami MGN. Ensino: as abordagens do processo. São Paulo: EPU, 1986.

9. Ecco I. Reflexões: temas do cotidiano escolar. Erechim, RS: Habilis, 2010

10. Freire P. Educação e Mudança. São Paulo: Paz e Terra; 1998.
11. Rocha CB, Correia GCS. Ética na docência do Ensino Superior. Revista Educare 2006;2:1-8.

12. Chrizostimo MM, Sanchez MCO, Xavier ML, Silvino ZR, Chrizostimo RM. 0 entrelace do perfil do professor com a formação profissional do enfermeiro com compromisso social. Rev Saúde Coletiva. 2019; 9(48):1393-98.

13. Severino AJ. Educação e universidade: conhecimento e construção da cidadania. Interface - Comunic, Saúde, Educ 2006;6(10):117-24.

14. Wild CFW, Nietsche E, Salbego C, Teixeira E, Favero NB. Validation of educational booklet: an educational technology in dengue prevention. Rev Bras Enferm. 2019;72(0):1318-25. DOI: http://dx.doi.org/10.1590/0034-7167-2018-0771.

15. Salbego C, Nietsche EA, Teixeira E, Girardon-Perlini NMO, Wild CF, Ilha S. Care-educational technologies: an emerging concept of the praxis of nurses in a hospital context. Rev Bras Enferm. 2018;71(6): 2666-74. DOI: http://dx.doi.org/10.1590/00347167-2017-0753.

16. Garcia Junior CAS, Verdi MIM. Dimensão dos Problemas éticos implicados na educação médica. Rev. bras. educ. med. [online]. 2019. 43(4) [acessado em 20 de junho de 2020]; 99-108. Disponivel em: http://www.scielo.br/scielo.php?script=sci_arttext\&pid=S0100-55022019000400099\&Ing=en\&nrm=iso

17. Bermamim MD, Prado P. Problematização do trabalho em equipe em enfermagem: relato de experiência. Rev. Bras Enfer 2013;66(1):134-137.

18. Chrizostimo MM, Brandão AAP. Perfil dos professores e formação profissional com

compromisso social: Estudo de caso. Revista Pró-UniverSUS. 2017 Jun./Dez.; 08 (2): 21-27 